\title{
Less hypoglycaemias in single room maternity care*
}

\author{
Peter Gerrits $^{1 \#,}$, Maartje de Hosson ${ }^{1}$, Ben Semmekrot ${ }^{1}$, Jan Sporken ${ }^{2}$ \\ ${ }^{1}$ Department of Paediatrics, Canisius-Wilhelmina Hospital, Nijmegen, The Netherlands \\ ${ }^{2}$ Department of Gynaecology and Obstetrics, Canisius-Wilhelmina Hospital, Nijmegen, The Netherlands \\ Email: " p.gerrits@cwz.nl
}

Received 24 May 2013; revised 26 June 2013; accepted 4 July 2013

Copyright (C) 2013 Peter Gerrits et al. This is an open access article distributed under the Creative Commons Attribution License, which permits unrestricted use, distribution, and reproduction in any medium, provided the original work is properly cited.

\begin{abstract}
In January 2006, the Canisius-Wilhelmina Hospital introduced the concept of Single Room Maternity Care (SMRC) by realizing 13 labour rooms for mother, infant and partner. Benefits of this new care concept not only include maternal satisfaction and increased staff satisfaction, but also significant health benefits for the neonate. Since the introduction of the concept, we registered a sharp decrease in the number of hypoglycaemias (from $15.6 \%$ in 2005 to $2.5 \%$ in 2009). Varying causes, such as successful breastfeeding and/or improved attachment between mother and infant may contribute to the decrease of hypoglycaemias.
\end{abstract}

Keywords: Hypoglycaemia; Neonate; Single Room Maternity Care

\section{BACKGROUND}

The goal of Family Centered Maternity Care is the delivery of high-quality healthcare while recognizing, focussing on, and adapting to both the physical and psychosocial needs of the childbearing woman, her family, and her newborn. This philosophy is based on the assumption that childbirth is a wellness, not an illness. Only minimal and selective interventions are applied when necessary [1].

In January 2006, the Canisius-Wilhelmina Hospital in Nijmegen, The Netherlands, introduced SRMC. Each woman's labour, birth care and newborn care are given in the same room under the same circumstances. Emphasis in this single room is on a non medical atmosphere for mother and child, providing restfulness, privacy and safety. An important feature of this concept is the capability of all involved caregivers to treat both the mother and the infant in the same room. If safe from a medical

\footnotetext{
${ }^{*}$ Competing interests and financial disclosures for all authors: None.
}

\#Corresponding author. point of view, the newborn infant will remain in the same room with the mother. Partners are allowed to stay in the same room as well, provided that activities and the room's entourage can be adapted to the mother and infant's needs to a certain extent. For example: medical equipment is integrated into a piece of furniture which will remain out of sight when not needed.

In the literature, several advantages of SRMC are reported [2]. Attachment between mother and child benefits from SRMC. Also, breastfeeding appeared to be more successful when mother and child remain in the same room together [3]. Janssen et al. [4] observed that this concept of care significantly increased patients' satisfaction. This might be due to several changes, such as a reduction of patient transfers and less transfer in nursing care. As a remarkable finding, nursing personnel appeared to be more satisfied with the new care concept as well [5]. The concept turns out to be cost-effective: the construction of the new maternity rooms did not exceed the budget and numbers of nursing personnel needed were lower than before [6]. The positive influence of this care concept on the mother's wellbeing is well described. However, literature is sparse on the effects on the newborn's health.

Neonatal hypoglycaemia is one of the most common problems in newborns. Transient asymptomatic hypoglycaemias in healthy neonates make part of the physiological adjustment to extra-uterine life. However, severe and long-lasting hypoglycaemias may lead to neurological symptoms [7-9]. Glucose screening is indicated in patients at risk for hypoglycaemia according to a protocol. In our hospital, these patients are divided into two groups: 1) patients with increased risk of developing hypoglycaemia, and 2) patients at severe risk of developing hypoglycaemia. In the first group, glucose measurements are routinely performed at 2 and 4 hours after birth, respectively. In the second group, blood glucose measurements are routinely performed at 1, 2 and 4 hours after birth, respectively. In all cases, blood samples 
are taken before a feeding.

In breastfed infants, intravenous glucose infusions are given in combination with intensively latching the infant, and no additional feedings are given, in order not to jeopardize the quality of breastfeeding.

We observed that the newly developed Family Centered Care concept resulted in a decreased incidence of hypoglycaemias needing intravenous glucose infusion. A retrospective analysis was performed to find out if this observation could be substantiated with numbers.

\section{PATIENTS AND METHODS}

We consulted our electronic database of patients' diagnoses to identify the amount of hospital admissions for neonatal hypoglycaemia. Data on inpatient and outpatient deliveries and the incidence of hypoglycaemias were acquired by retrospective study of patient files from the years 2003-2009. Blood glucose determinations were carried out bed-side with the HemoCue B-glucose system, which was validated earlier by our laboratory.

Inpatient deliveries are defined as hospital deliveries managed by the obstetrician, whereas outpatient deliveries are hospital deliveries managed by hospital midwifes. As a definition for neonatal hypoglycaemia we adhered to the nationally and internationally used definition of $2.5 \mathrm{mmol} / \mathrm{l}$. Since the HemoCue B system, used for bed-side glucose determination, is a whole blood method, underestimating glucose concentrations with $10 \%-15 \%$, $2.2 \mathrm{mmol} / \mathrm{l}$ was used as the cut-off value. The same definition was used throughout the last decade.

\section{RESULTS}

Data analysis showed a clear decrease of the number of infants with hypoglycaemia after the maternity suites had been opened in January 2006. Simultaneously, the number of deliveries in the Canisius-Wilhelmina Hospital increased (2005: 1522 deliveries, 2008: 1790 deliveries). In relation to the total amount of deliveries, the number of newborns with hypoglycaemia dropped from $15.6 \%$ in 2005 to $2.7 \%$ in 2008.

In 2009, we observe a further decrease in the number of neonatal hypoglycaemias: 46 hypoglycaemias on a total number of 1875 deliveries (2.5\%).

These numbers suggests a clear correlation between the introduction of the novel SRMC concept and the incidence of neonatal hypoglycaemia.

\section{DISCUSSION}

Special attention to breastfeeding is present in our hospital for more than 10 years. In 2004, we introduced in our hospital a new breastfeeding protocol according to the WHO and Unicef Baby-Friendly Hospital Initiative (BFHI), and simultaneously adapted the hypoglycaemia- protocol. No changes in the approach of breastfeeding and hypoglycaemias occurred since then, apart from the introduction of the novel SRMC concept. Furthermore, there were no changes in supplemental feeds given to infants who were not with the mother, and there were no changes in the incidence of IDM and LGA and SGA births during the study period. Since the introduction of the SRMC concept, we registered a sharp decrease in the number of neonatal hypoglycaemias. Multiple factors might be responsible for the decrease in neonatal hypoglycaemias, such as successful breastfeeding and improved attachment between mother and infant. Immediately after birth, skin-to-skin contact between mother and infant facilitates breastfeeding. Since mother and infant stay close together, the mother is close to respond to her infant's signals. Indeed, this provides on demand feeding and stimulates mothers' early milk production [10]. The involvement of a single nurse in the total care process for both mother and infant may also contribute to the success of breastfeeding: there is less transfer of care, less transport, and therefore less stress for mother and infant.

Benefits of the new care concept are not only maternal satisfaction and increased staff satisfaction, but apparently also measurable health benefits for the neonate. This is an interesting development. In October 2008 we introduced single-room neonatal care, also based on the Family Centered Care principles [11,12], a novelty in Europe at that time. The latter development enables us to quantify the success rates of breastfeeding prior to and after the introduction of this concept of care. At the moment we are busy doing so.

Of course, a methodological limitation is the retrospective character of our study. We observe an association, not a proven cause and effect relation. We strongly feel the importance, however, of reporting the spectacular decrease in neonatal hypoglycaemias we observed. It suggests that the impact of the problem of neonatal hypoglycaemia can be reduced by going back to nature, allowing newborn infants to stay with their mothers for optimal adaptation of their glucose homeostasis.

To conclude, this observation shows that there is a clear correlation between the introduction of a new concept of care and a lower incidence of hypoglycemias in newborns, as a consequence of an increased mother-infant interaction. In our opinion, this is an important observation, worth reporting to stimulate future research on this topic.

\section{REFERENCES}

[1] Zwelling, E. and Phillips, C.R. (2001) Family-centered maternity care in the new millennium: Is it real or is it imagined? The Journal of Perinatal \& Neonatal Nursing, 15, 1-12. doi:10.1097/00005237-200112000-00002 
[2] Hunter, M.A. and Larrabee, J.H. (1988) Women’s perceptions of quality and benefits of postpartum care. Journal of Nursing Care Quality, 13, 21-30. doi:10.1097/00001786-199812000-00004

[3] Pérez-Escamilla, R., Pollit, E., Lonnerdal, B. and Dewey, K.G. (1994) Infant feeding policies in maternity wards and their effect on breast-feeding success: An analytical overview. American Journal of Public Health, 84, 89-97. doi:10.2105/AJPH.84.1.89

[4] Janssen, P.A., Klein, M.C., Harris, S.J., Soolsma, J. and Seymour, L.C. (2000) Single room maternity care and client satisfaction. Birth, 27, 235-243. doi:10.1046/j.1523-536x.2000.00235.x

[5] Janssen, P.A., Harris, S.J., Soolsma, J., Klein, M.C. and Seymour, L.C. (2001) Single room maternity care: The nursing response. Birth, 28, 173-179. doi:10.1046/j.1523-536x.2000.00235.x

[6] Harris, S.J., Farren, M.D., Janssen, P.A., Klein, M.C. and Lee, S.K. (2004) Single room maternity care: Perinatal outcomes, economic costs and physician preferences. Journal of Obstetrics and Gynaecology Canada, 26, 633-640.

[7] Nicholl, R. (2003) What is the normal range of blood glucose concentrations in healthy term newborns? Ar- chives of Disease in Childhood, 88, 238-239. doi:10.1136/adc.88.3.238

[8] Hoseth, E., Joergensen, A., Ebbesen, F. and Moeller, M. (2000) Blood glucose levels in a population of healthy, breast fed, term infants of appropriate size for gestational age. Archives of Disease in Childhood-Fetal and Neonatal Edition, 83, F117-119. doi:10.1136/fn.83.2.F117

[9] Heck, L.J. and Erenberg, A. (1987) Serum glucose levels in term neonates during the first 48 hours of life. Journal of Pediatrics, 110, 119-122. doi:10.1016/S0022-3476(87)80303-7

[10] Yamauchi, Y. and Yamanouchi, H. (1990) The relationship between rooming-in/not rooming-in and breast-feeding variables. Acta Paediatrica Scandinavica, 79, 10-22. doi:10.1111/j.1651-2227.1990.tb11377.x

[11] Voeten, M., Gerrits, G.P.J.M., Voorhoeve, P.G. and Semmekrot, B.A. (2008) Treatment of neonatal hypoglycaemia: More frequent latching versus supplementary feeding with formula; retrospective study of patient files. Nederlands Tijdschrift voor Geneeskunde, 152, 1732 1736.

[12] White, R.D. (2003) Individual rooms in the NICU-An evolving concept. Journal of Perinatology, 1, S22-S24.

\section{ABBREVATIONS}

SRMC: single room maternity care 